\title{
Infectious Mononucleosis with Upper Airway Obstruction: when tracheotomy and tonsillectomy can be helpfull
}

\section{Enrico Maria Amadei ${ }^{1}$, Laura Benedettini ${ }^{2}$ and Marco Trebbi ${ }^{1}$}

1Department of Otorhinolaryngology and Audiology, Infermi Hospital, Rimini, Italy.

${ }^{2}$ Department of Transfusion Medicine and Immunohematology, Infermi Hospital, Rimini, Italy.

Corresponding Author: Enrico Maria Amadei, Department of Otorhinolaryngology and Audiology, Infermi Hospital, Rimini, Italy.

Received Date: February 26, 2021; Accepted Date: April 05, 2021; Published Date; April 08,2021

Citation: Enrico M. Amadei, Benedettini L. and Trebbi M. (2021) Infectious Mononucleosis with Upper Airway Obstruction: when tracheotomy and tonsillectomy can be helpful. J. Clin otorhin. 3(2); DOI:10.31579/2692-9562/028

Copyright: (C) 2021 Enrico Maria Amadei, This is an open-access article distributed under the terms of the Creative Commons Attribution License, which permits unrestricted use, distribution, and reproduction in any medium, provided the original author and source are credited.

\section{Abstract}

Infectious mononucleosis is caused by Epstein-Barr virus, which infects more than $98 \%$ of the world's adult population. Approximately $90 \%$ of adults become antibody-positive before the age of 30 . The ages of $15-24$ years are the most likely time for the onset of symptoms.

We report the case of a 13-year-old girl who came to our emergency department for bilateral erythematouspoltaceous tonsillitis complicated by dysphagia and dyspnea. She had a diagnosis of infectious mononucleosis.

For some hours the young woman presented an inconsistency between a marked respiratory distress and the patency of her upper airways. Finally the clinical picture fell suddenly, requiring an emergency tracheotomy to ensure a patent airway. In the following days a diagnosis of a left parapharyngeal abscess was reached. A tonsillectomy with drainage of the parapharyngeal abscess was required.

It is well known that infectious mononucleosis is typically a silent infection. We describe the case of a girl who risked losing her life due to aggressive infectious mononucleosis, and how we treated her. Such a complicated case has never been described in Literature.

Keywords: infectious mononucleosis; complications; airway obstruction; parapharyngeal abscess; tracheotomy; tonsillectomy

Subtitle: Infectious Mononucleosis generally, but not always, is a silent infection

\section{Introduction}

Infectious mononucleosis (IM) is caused by Epstein-Barr virus (EBV), which infects more than $98 \%$ of the world's adult population. Approximately $90 \%$ of adults become antibody-positive before the age of 30. The age at which primary EBV infection is acquired increases in developed Countries [1]. The incidence in the community of IM is $45 / 100000$, but among adolescents is about 350/100000 [2]. This infection is usually asymptomatic in childhood. The ages of 15-24 years are the most likely time for the onset of symptoms [3]. Patients classically complain fever, fatigue and malaise, lymphadenopathy and odynophagia which resolve within a few weeks.

Complications of IM are numerous but generally rare, such as neurologic, hematologic, cardiac, psychological complications, hepatitis. Some laboratory manifestations are well known [2], such as neutropenia (50$80 \%$ ), lymphocytosis (more than $4,000 \mathrm{~mm}^{3}$ ), anemia (3\%), thrombocytopenia (25-50\%), asymptomatic elevated transaminases (50$80 \%)$. Ampicillin rush is also frequent $(50-95 \%)$, i.e. the erythematous rash on the body, resulting from the intake of beta-lactams in subjects suffering from IM. Interestingly, all doctors are scared of the splenic rupture, which is a rare occurrence $(<0.5 \%)$ and to prevent this it is enough to avoid strain for 3 weeks [1]. On the contrary, many doctors do not know that neurological disorders, and especially the airway obstruction are not so rare complications of IM $(\leq 5 \%)$ [2].

The purpose of this paper is to present a case of complicated IM, which endangered the life of a young girl, and how we managed this situation. Such a case has never been reported before in Literature.

\section{Case report}

We present the case of a previously healthy 13-year-old girl who came to our emergency department due to persistent dysphagia and odynophagia for 5 days, despite oral betamethasone $5 \mathrm{mg} 2$ times a day. She exhibited mild fever $\left(37.2^{\circ} \mathrm{C}\right)$, exudative obstructive tonsillar hypertrophy and marked bilateral cervical lymphadenopathy. She had slight dyspnea, without tirage or cornage. Oxygen saturation was $98 \%$ in ambient air. The blood tests showed positive IgM against Viral Capsid Antigen (VCA) of $\operatorname{EBV}(160 \mathrm{U} / \mathrm{ml})$, normal white blood cells in number $(6.720 / \mathrm{mmc})$, with 
lymphocytosis (48.5\%), Glutamic Pyruvic Transaminase (GPT) was 79 $\mathrm{U} / \mathrm{L}$.

Anti-Toxoplasma IgM and IgG were negative, anti-CMV IgG positive and anti-CMV IgM negative, anti-Mycoplasma pneumoniae IgM negative. An otorhinolaryngologist excluded a peritonsillar abscess. Flexible rhinofibroscopy revealed a preserved respiratory space. An ultrasound of the neck revealed marked bilateral lymphadenopathies and excluded signs of colliquation.

After 6 hours the patient presented an increased speech difficulty, with snoring breathing. She had a forced sitting position, even without an increase in respiratory rate (18 breaths per minute). No tirage or cornage, no desaturation. The indication was given for admission to a pediatric ward and intravenous cortisone therapy was initiated (methylprednisolone $1 \mathrm{mg} / \mathrm{kg}$ every 6 hours).

A few hours later the girl suddenly began to desaturate, losing consciousness. An ineffective oral and naso-tracheal intubation required an emergency percutaneous tracheotomy, to stabilize the patient and to ensure effective ventilation. Because of a right pneumothorax she also underwent a thoracotomy.

The following day a surgical tracheotomy was performed (Fig 1). An exudative obstructive hypertrophy of the palatine and lingual tonsils was found, with a diffuse stenosing edema of the oro-hypopharynx. Some biopsies were performed on these anatomical regions for diagnostic purposes, to rule out a lymphoproliferative disease.

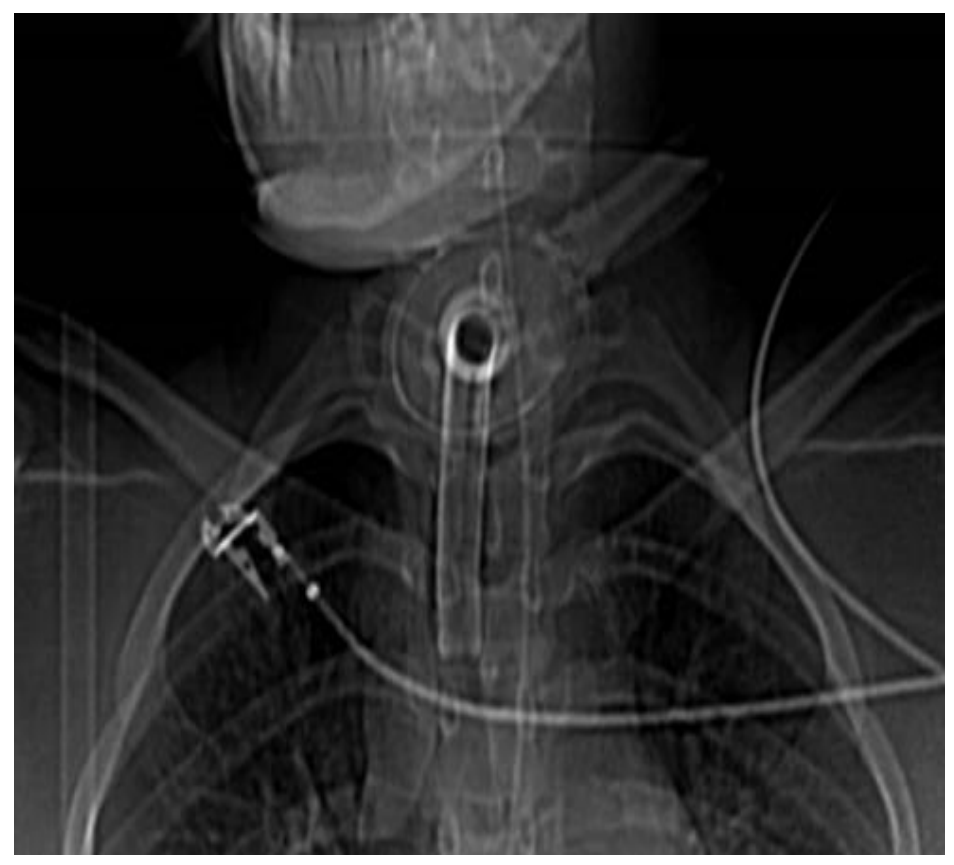

Figure1: Surgical tracheotomy, carried out to ensure good ventilation.

Flexible rhinofibroscopy was repeated daily. After 8 days it again revealed a complete upper airways obstruction above the glottic plane, with epiglottic edema and diffuse tonsillar necrosis. Finally a cervical CT showed a left parapharyngeal abscess and a diffuse colliquation of cervical lymph nodes (Figure 2-3).

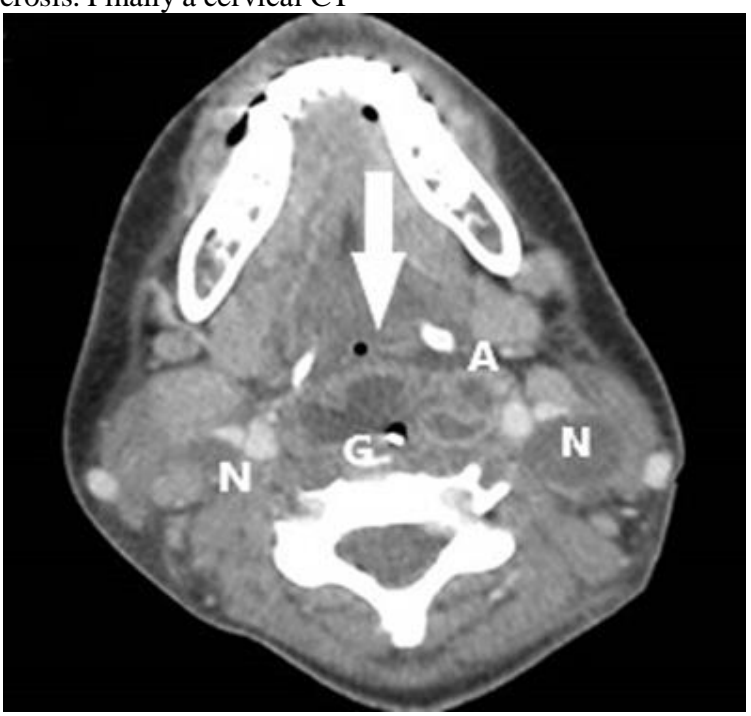

Figure 2: CT shows a complete obstruction of the upper airways, with diffuse pharyngeal edema (arrow), left parapharyngeal abscess (A) and 


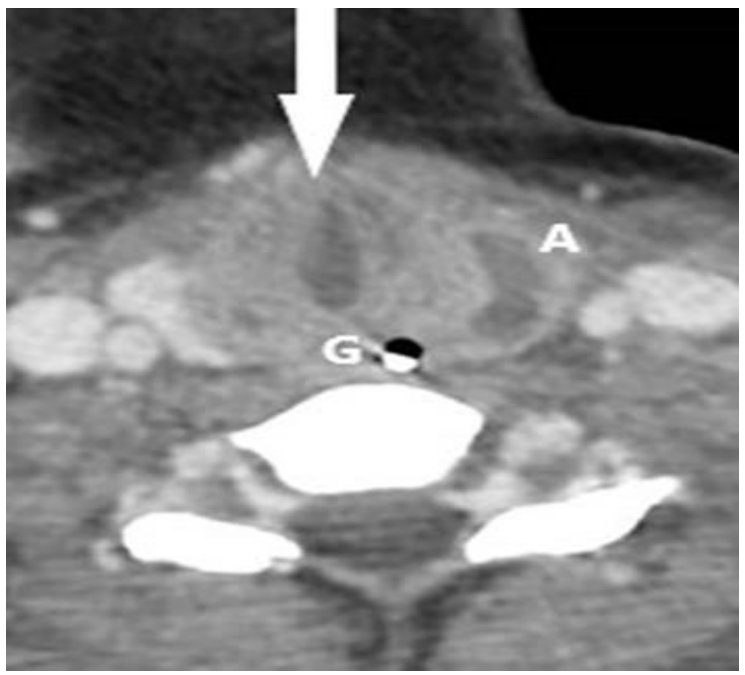

Figure 3: CT shows a complete obstruction of the upper airways, with diffuse laryngeal edema (arrow) and left parapharyngeal abscess (A). Nasogastric tube $(G)$.

2 days later a bilateral pericapsular tonsillectomy was performed, with drainage of the abundant purulent collection from the lower pole of the left tonsillar lodge.

After an infectious disease consultation, antibiotic therapy with meropenem plus clindamycin was administered. These were discontinued after the onset of an erythematous skin rash and because of absence of elevated indices of inflammation.

The young woman was transferred from reanimation to the pediatric department after 17 days of hospitalization. She presented the need for tracheal cannula for 1 month, due to the persistence of the upper airway obstruction. She gradually recovered her speaking and swallowing skills. She was released at home after a total of 38 days of hospitalization, with continuation of speech therapy rehabilitation.

\section{Discussion}

Transmission of IM occurs mainly with saliva. Kissing is the major route of transmission of EBV infection among adolescents and young adults, while children transmit IM between each other by exchanging infected toys or contract it by their infected parent. Incubation period of IM is about 6 weeks [1]. Primary infection in childhood is lower in areas with better sanitary conditions. In young adults, the rate of developing IM from primary EBV infection is estimated at 50\% [4]-75\% [1].

The differential diagnosis should be placed with infections caused by Cytomegalovirus, Toxoplasma gondii, Human Immunodeficiency Virus, Adenovirus, etc. [2].

The simplest and cheapest way to confirm the clinical diagnosis is to search for heterophile antibodies. These are IgM class antibodies, and they are directed against mammalian erythrocytes. This test has a sensitivity of $85 \%$ ( $72 \%$ the first week), but it is aspecific and it could be persistent negative in young children. The search for VCA IgM antibody is more expensive, but specific and it has a positivity of $95 \%$ (85\% the first week). These antibodies become negative 3-12 months post infection [1].

It is well known that IM is typically a silent infection and that vast majority of adult population has antibodies against EBV, without remembering ever being infected [1-2]. This infection is usually asymptomatic in childhood. The ages of 15-24 years are the most likely time for the onset of symptoms [3]. Patients classically complain fever, fatigue and malaise, lymphadenopathy and odynophagia [1-4].

Complications of IM are rare. Asymptomatic elevated transaminases are found in 50-80\% of cases, while jaundice is present in 5\% of patients [2]. Strangely, well known is the splenic rupture, which is certainly not the most frequent $(<0.5 \%)$. Many doctors underestimate the frequency of neurological disorders $(1-5 \%)$, such as neuritis, encephalitis, cerebellitis, transverse myelitis, Guillain-Barrè syndrome.

Upper airway obstruction (UAO), secondary to IM, is a not rare complication $(<5 \%)$. It is a potentially lethal occurrence and it is one of the most common indications for hospitalization from IM [2]. UAO can have many different causes, which can be more or less frequent [5-8].

About the patient of this paper, two conditions were potentially lethal: an exudative obstructive hypertrophy of the palatine and lingual tonsils, with a diffuse edema of the oro-hypopharynx; and a left parapharyngeal abscess, with persistent airway impairment. Such a complicated case has never been described in Literature until now. UAO is characterized by cornage, tirage, use of accessory respiratory muscles, tachypnea and cyanosis. Desaturation is often late, but unfortunately it is sudden. In a study by Kilham et al. objectivable signs were sought to quantify respiratory distress and predict desaturation. They concluded that eyes of an experienced observer about child's general appearance were far more valid than any measurable data [9].

Generally UAO post-IM is managed with early intubation of young patients and sometimes with tonsillectomy in Literature. Lots of case reports about tracheotomies performed because of UAO resulting from IM were published between the 40s and 80 s of the twentieth century [68]. In those years the term mononucleosis was not used, but this disease was known as "anginose type of glandular fever" or as "the kissing disease"[3]. It seems incredible that an emergency tracheotomy was needed in 2021 . Yet tracheotomy proved to be, once again, the only quick and safe method to resolve a rapidly evolving UAO, if ineffective intubation.

Some Authors consider tonsillectomy the most effective way to resolve the lasting obstruction $[10,11]$. There is no agreement between Authors on surgery, as the risk of postoperative bleeding is high [12]. Lloyd proposed to resort to intracapsular tonsillectomy with microdebridment, with good results [13]. We are not used to perform a tonsillectomy, as 
long as the acute inflammation persists, because the risk of postoperative bleeding, even if some papers deny this increased risk [14-15]. However, in this case, this procedure was inevitable. It allowed us to resolve a persistent obstruction of the upper airways, caused by the hypertrophy of the tonsillar lymphatic tissue and by a left parapharyngeal abscess.

In symptomatic cases of IM with fever, malaise and pharyngodynia, usually only medicines for fever and pain are used. In Literature there is not uniformity about the use of cortisone for symptom control of IM [3]. This on one hand would reduce inflammation and edema, on the other hand it could prolong the duration of symptoms, also favouring the onset of complications. Some Authors believe that the use of high-dose cortisone (intravenous dexamethasone $0.25 \mathrm{mg} / \mathrm{kg}$ or methylprednisolone $1 \mathrm{mg} / \mathrm{kg}$ every 6 hours) in case of UAO is indicated and essential $[2,16]$. We believe that the use of high-dose cortisone is the only really useful medical therapy in case of UAO.

Although we know that IM is a viral infection, we tried to prevent even greater complications, such as parapharyngeal abscess, due to the appearance of a neutrophilic leukocytosis. Womack et al. recommend using antibiotics only in case of positive rapid test for group A $\beta$ haemolytic streptococcus pharyngitis [4]. In this specific case, not only cortisone did not reduce the edema, but even antibiotics did not prevent the abscess. We stopped them at the onset of a widespread skin rash. So in our experience, although we typically use cortisone and antibiotics, both have proved ineffective with our young patient. We do not use antivirals for IM, as there is lack of evidence regarding their effectiveness [2].

We have still many doubts about IM. It is not known why some young people become asymptomatically infected and others develop a severe and potentially fatal disease. It is not know why in some cases autoimmune diseases or malignancies develop following EBV infection. Certainly a vaccine against EBV would be useful, but it is not yet available to date. We hope that the big push in virus vaccination research, such as the recent Sars-Cov2 race, can help prevent future EBV infection and complications.

\section{Conclusion}

IM is a very common but often asymptomatic infection. The symptomatic forms are more common among adolescents and young adults. Complications are rare, but not to be forgotten. Upper airway obstruction can be sudden and life-threatening for these patients. Tracheotomy and tonsillectomy are surgical procedures that can solve even the most desperate cases.

Conflict of Interest: None to declare.
Statement of Ethics: The parents gave their informed consent to write this paper.

\section{References:}

1. Dunmire S. (2015). Infectious Mononucleosis. Curr Top Microbiol Immunol; 390: 211-240.

2. Jenson HB. (2000). Acute complications of Epstein-Barr virus infectious mononucleosis. Curr Opin Pediatr;12: 263-268.

3. Rezk E, Nofal YH, Hamzeh A, Aboujaib MF, AlKheder MA, et.al. (2015). Steroids for symptom control in infectious mononucleosis. Cochrane Database of Systematic Reviews;11.

4. Womack J, Jimenez M. (2015). Common questions about infectious mononucleosis. Am Fam Physician; 91(6): 372-376.

5. Amadei EM, Benedettini L, Piccin O. (2014). Two Cases of Cervical Hemorrhage with Upper Airway Obstruction: A Life-Threatening Condition. Case Reports in Medicine;

6. Jones GP, Jones E. (1949). Anginose type of glandular fever requiring tracheotomy. $\mathrm{Br}$ Med $J$; 2(4638): 1212.

7. Carrington P, Hall JI, (1986). Fatal airway obstruction in infectious mononucleosis. Br Med J (Clin Res Ed); 292(6514): 195.

8. Story GBT, MacCabe AF. (1946). A Case of Infectious Mononucleosis Requiring Tracheotomy. Br Med J; 2(4478).

9. Kilham H, Gillis J, Benjamin B. (1987). Severe upper airway obstruction. Pediatr Clin North Am; 34: 1-14.

10. Windfuhr J, Toepfner N, Steffen G, Waldfahrer F, Berner R. (2016). Clinical practice guideline: tonsillitis II. Surgical management. Eur Arch Otorhinolaryngol; 273(4): 989-1009.

11. Chan SC, Dawes PJ. (2001). The management of severe infectious mononucleosis tonsillitis and upper airway obstruction. J Laryngol Otol; 115(12): 973-977.

12. Giger R, Landis BN, Dulguerov P. (2005). Haemorrhage risk after quinsytonsillectomy. Otolaryngol Head Neck Surg; 133: 729-734.

13. Lloyd AM, Reilly BK. (2020) Infectious Mononucleosis and Upper Airway Obstruction: Intracapsular Tonsillectomy and Adenoidectomy With Microdebrider for Prompt Relief. Ear Nose Throat J; 145561320930046.

14. Slouka D, Čejková Š, Hanáková J, Hrabačka P, Kormunda S, et.al (2021). Risk of Postoperative Bleeding in Tonsillectomy for Peritonsillar Abscess, as Opposed to in Recurrent and Chronic Tonsillitis-A Retrospective Study. Int J Environ Res Public Health; 18(4):1946.

15. G Lehnerdt, K Senska, K Jahnke, M Fischer. (2005). Posttonsillectomy haemorrhage: a retrospective comparison of abscessand elective tonsillectomy. Acta Otolaryngol; 125(12): 1312-131

16. Glynn FJ, Mackle T, Kinsella J. (2007). Upper airway obstruction in infectious mononucleosis. Eur J Emerg Med; 14(1): 41-42.

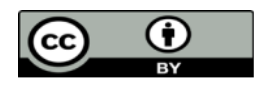

This work is licensed under Creative Commons Attribution 4.0 License

To Submit Your Article Click Here: Submit Manuscript

DOI: $10.31579 / 2692-9562 / 028$
Ready to submit your research? Choose Auctores and benefit from:

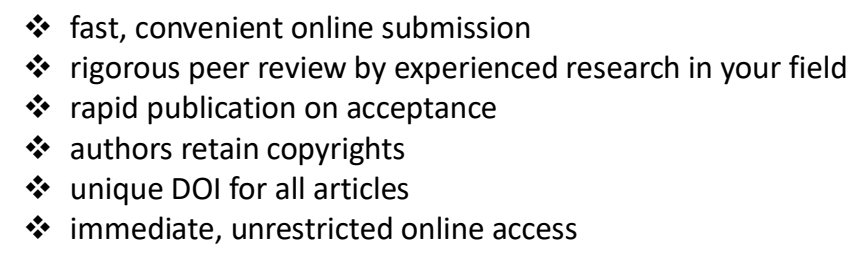

At Auctores, research is always in progress.

Learn more www.auctoresonline.org/journals/journal-of-clinicalotorhinolaryngology 